\title{
Identification of MCI Using Optimal Sparse MAR Modeled Effective Connectivity Networks
}

\author{
Chong-Yaw Wee ${ }^{1, *}$, Yang Li ${ }^{1,2, *}$, Biao Jie ${ }^{1,3}, \mathrm{Zi}^{-W e n}$ Peng $^{1}$, and Dinggang Shen ${ }^{1}$ \\ ${ }^{1}$ Department of Radiology and BRIC, \\ University of North Carolina at Chapel Hill, NC, USA \\ ${ }^{2}$ Beihang University, Beijing, China \\ ${ }^{3}$ Department of Computer Science and Engineering, \\ Nanjing University of Aeronautics and Astronautics, Nanjing, China \\ dgshen@med.unc . edu
}

\begin{abstract}
Capability of detecting causal or effective connectivity from restingstate functional magnetic resonance imaging (R-fMRI) is highly desirable for better understanding the cooperative nature of the brain. Effective connectivity provides specific dynamic temporal information of R-fMRI time series and reflects the directional causal influence of one brain region over another. These causal influences among brain regions are normally extracted based on the concept of Granger causality. Conventionally, the effective connectivity is inferred using multivariate autoregressive (MAR) modeling with default model order $q=1$, considering low frequency fluctuation of R-fMRI time series. This assumption, although reduces the modeling complexity, does not guarantee the best fitting of R-fMRI time series at different brain regions. Instead of using the default model order, we propose to estimate the optimal model order based upon MAR order distribution to better characterize these causal influences at each brain region. Due to sparse nature of brain connectivity networks, an orthogonal least square (OLS) regression algorithm is incorporated to MAR modeling to minimize spurious effective connectivity. Effective connectivity networks inferred using the proposed optimal sparse MAR modeling are applied to Mild Cognitive Impairment (MCI) identification and obtained promising results, demonstrating the importance of using optimal causal relationships between brain regions for neurodegeneration disorder identification.
\end{abstract}

\section{Introduction}

Mild cognitive impairment (MCI), an intermediate state of cognitive function between normal aging and dementia [1], has gained a great deal of attention recently due to its high progression rate to Alzheimer's disease (AD). Exploring structural and functional interactions among various brain regions enable us to better understand the pathological underpinnings of MCI. These interaction patterns, which can be characterized via connectivity networks, have been applied recently to disease identification. Functional interactions among brain regions can be extracted from functional magnetic

\footnotetext{
* The authors contribute equally to this work. 
resonance imaging (fMRI). However, functional connectivity, which is derived from the traditional correlation analysis, is non-directional and is unable to adequately account for the functional interactions between different brain regions. To remedy this problem, the brain connectivity derived based on the concept of Granger causality has been suggested to explore the causal relationships of fMRI time series [2]. Granger causality investigates whether prediction of the present value of one time series by its own past can be improved by including the past courses of other time series [3]. This causality-based directional connectivity is referred as effective connectivity in the literature.

Effective connectivity can be efficiently estimated from fMRI time series using multivariate autoregressive (MAR) modeling [4]. In traditional MAR modeling, fMRI time series is modeled using default model order $(q=1)$ due to low frequency fluctuations of fMRI signals. However, this default model order based modeling does not guarantee the best fitting of fMRI time series at different brain regions. In this paper, we propose a sparse MAR modeling to infer effective connectivity networks using the optimal orders estimated based on probabilistic distribution of model order. Orthogonal least square (OLS) algorithm is further incorporated to preserve only a small number of regressors in MAR modeling, minimizing possible spurious connectivity. Promising results on MCI identification demonstrate the superiority of the proposed approach, compared to both the correlation-based functional connectivity and the default model order based effective connectivity approaches.

\section{Materials and Methodology}

\section{$2.1 \quad$ Dataset}

Twelve MCI individuals (6 males and 6 females) and 25 socio-demographically matched normal controls ( 9 males and 16 females) were included in this study. The mean ages in terms of year for MCI and control groups are 75.0 \pm 8.0 and 72.9 \pm 7.9 , respectively. All the participants were scanned using a 3.0 Tesla scanner with the following parameters: repetition time $(\mathrm{TR})=2000 \mathrm{~ms}$, echo time $(\mathrm{TE})=32 \mathrm{~ms}$, flip angle $=77^{\circ}$, acquisition matrix $=64 \times 64$, voxel size $=4 \mathrm{~mm}$. One hundred fifty resting-state fMRI (R-fMRI) volumes were acquired. Participants were requested to keep their eyes open and stared at a fixation cross during scanning.

\subsection{Data Preprocessing}

R-fMRI images were preprocessed using Statistical Parametric Mapping software package (SPM8). Specifically, the first $10 \mathrm{R}$-fMRI volumes were removed before parcellating the brain space into 116 regions-of-interest (ROIs) based on the automated anatomical labeling (AAL) atlas [5]. The mean R-fMRI time series was then computed for each subject by averaging the R-fMRI time series over all voxels in each ROI. Prior to effective connectivity network construction, regression of nuisance signals including white matter and cerebrospinal fluid (CSF) signals, and six headmotion profiles was performed on R-fMRI time series. Global signal regression is not performed due to its controversies in the R-fMRI preprocessing steps [6]. Note that 
there is no significant difference in terms of head-motion profiles between the MCI and control groups ( $p>0.218$ in any direction).

\subsection{Motivations}

In this study, we choose to use MAR modeling to infer effective connectivity networks of cortical activity for two reasons. First, MAR model is a dynamic model that can capture the temporal information of all possible combinations of region pairs in the model. Second, this model is efficient in measuring the directional influences between brain regions based on the concept of Granger causality [7, 8].

In conventional MAR modeling, R-fMRI time series is normally modeled using a default model order, i.e., $q=1$ [9]. However, this default model order does not guarantee to provide the best fitting of R-fMRI time series at different brain regions since they might interact differently. Furthermore, it has been suggested that functional interactions among ROIs are affected by disease [10]. Thus, it is important to determine the optimal model order that best fits the R-fMRI time series of each ROI in different clinical groups. Accordingly, we propose to model the R-fMRI time series of each ROI using its optimal model order. We hypothesize that this optimal MAR modeling can better characterize the time series in each ROI, and potentially be used to improve patient-control identification accuracy.

Due to the sparse nature of brain networks, we incorporate the orthogonal least square (OLS) algorithm into MAR modeling to minimize spurious connections and physiological noise caused by cardiac and respiratory cycles. Specifically, we utilize OLS algorithm to identify the significant regressors in MAR model by measuring the direct interaction strength between regions based on the estimated optimal model order. It has been shown that a satisfactory sparse MAR model for best fitting the fMRI time series can be obtained with these procedures [11].

\subsection{MAR Modeling for Effective Connectivity}

Suppose we have $M$ time series generated from $M$ ROIs within a system of $N$ RfMRI volumes, i.e., $y(t)=\left[y_{1}(t), y_{2}(t), \cdots, y_{M}(t)\right]^{T} \in \mathbb{R}^{M \times 1}$ with $(t=1,2, \cdots, N)$, the $q$-th order MAR model predicts the next value of the $M$-dimensional time series, $y(t)$, using a linear combination of $q$ previous vectors as

$$
y(t)=\sum_{i=1}^{q} A(i) y(t-i)+E(t)
$$

where $A(i) \in \mathbb{R}^{M \times M}(i=1,2, \cdots, q)$ is the MAR coefficients matrix and $E(t)=$ $\left[e_{1}(t), e_{2}(t), \cdots, e_{M}(t)\right]^{T}$ is the residual vector. If $q=0$, the MAR model can be simplified to the linear correlation of current measurements among brain regions. Eq. (1) can be rewritten as the standard form of multivariate linear regression mode as

$$
y(t)=\varphi(t) \theta+E(t)
$$

where $\varphi(t)=[y(t-1), y(t-2), \cdots, y(t-q)]$ is the $q$ previous multivariate time series and $\theta$ is a $(q \times M) \times M$ matrix of MAR coefficients or weights. If the $n$-th 
row of $Y, X$, and $E$ (given below) are $y_{n}(t), \varphi_{n}(t)$, and $e_{n}(t)$, respectively, we can then recast the dynamics of ROIs of the network as a multivariate regression model as

$$
Y=X \theta+E,
$$

where $Y$ is a $(N-q) \times M$ matrix, $X$ is a $(N-q) \times(q \times M)$ matrix, $\theta$ is a $(q \times M) \times M$ matrix, and $E$ is a $(N-q) \times M$ matrix. Each row of $Y$ in Eq. (3) corresponds to a typical scan of fMRI data, while columns indicate the time series of each region. Thus, MAR model quantifies the linear dependence of one brain region upon all other brain regions, where every element in $\theta$ matrix can be interpreted as the influence from all the brain regions. Dependence between pair of brain regions is reflected by a nonzero magnitude, while independence leads to a zero element in matrix $\theta$.

\subsection{Optimal Sparse MAR Modeling and Effective Connectivity}

In this study, Bayesian information criterion (BIC) [12] is used to determine the optimal MAR model order of each ROI. Specifically, model order $q$ can be estimated by minimizing the BIC value as

$$
\operatorname{BIC}(q)=N \log [\operatorname{mse}(q)]+q \log (N),
$$

where mse is the mean square error or the residual sum of squares. A range of $q=[1,2, \cdots, 10]$ is normally used to search for the optimal model order. Then, statistical $t$-test is used to explore differences of model order distribution between patient and control groups. In this way, we can identify the brain regions with significant different MAR model order between patients and normal controls.

It is unlikely that any of the components in $\theta$ matrix is exactly zero. To achieve sparse MAR modeling, OLS regression algorithm is used to statistically test if the entries in $\theta$ are vanishing. In the OLS algorithm, error reduction ratio (ERR) criterion, which is equivalent to the squared correlation coefficient, is used to measure the significance of the candidate regressors (elements in $\theta$ ). The effective connectivity between region $m 1$ with time series $x_{m 1}=\left[y_{m 1}(1), y_{m 1}(2), \cdots, y_{m 1}(N)\right]^{T}$ and region $m 2$ with time series $x_{m 2}=\left[y_{m 2}(1), y_{m 2}(2), \cdots, y_{m 2}(N)\right]^{T}$ is defined as [11]

$$
C\left(x_{m 1}, x_{m 2}\right)=\frac{\left(\left(x_{m 1}\right)^{T} x_{m 2}\right)^{2}}{\left(\left(x_{m 1}\right)^{T} x_{m 1}\right)\left(\left(x_{m 2}\right)^{T} x_{m 2}\right)}=\frac{\left(\sum_{t=1}^{N} x_{m 1}(t) x_{m 2}(t)\right)^{2}}{\sum_{t=1}^{N}\left(x_{m 1}(t)\right)^{2} \sum_{t=1}^{N}\left(x_{m 2}(t)\right)^{2}} .
$$

Similar to the commonly used Pearson correlation coefficient, Eq. (5) reflects the linear relationship between vectors $x_{m 1}$ and $x_{m 2}$. Based on the estimated optimal model order, we can obtain the optimal sparse MAR model, which will be used to infer the optimal effective connectivity matrix, $C$, of size $M \times M$.

\subsection{Effective Connectivity Matrix for Disease Identification}

We perform two-sample two-tailed $t$-tests to identify $d$ regions that are significantly different ( $p<0.05$, corrected) between MCI and control groups in terms of their model order distributions. Based on the determined model orders, we explore the 
causal relationships of these $d$ regions with other brain regions. Specifically, for MCI group with 12 subjects and optimal order $q=q_{1}$, we build an effective connectivity matrix of size $12 \times 116$ for each of $d$ regions. One sample $t$-test is then used to determine the relationships between each $d$ region with all other regions. The same procedures are repeated for the control group with 25 subjects and the optimal order $q=q_{2}$. Note that, if $q>0$, there are causal relationships between brain regions, while there are only correlation relationships when $q=0$.

For each subject, effective connectivity matrix of size $d \times 116$ is constructed for $q=q_{1}$ and $q=q_{2}$. (Note that $q_{1}$ and $q_{2}$ are the optimal orders for MCI and control groups, respectively.) These two effective connectivity matrices are then stacked to form a new connectivity matrix $W$ of size $(2 \times d) \times 116$. Elements in the connectivity matrix $W$ are concatenated into a long feature vector to represent each subject, and then a subset of the most discriminative features is selected using the support vector machine recursive feature elimination (SVM-RFE) algorithm [13]. The selected features will be used for disease classification.

\section{Results}

It is observed that the optimal MAR model orders, $q$, for different ROIs are either 0 or 1. This implies that some ROIs are linearly correlated with other regions, while other ROIs are causally influenced by other regions. Significant different model order distribution is observed between MCI and control groups in four regions, i.e., middle and posterior cingulate gyri (MCG and PCG), lingual gyrus (LING), and caudate (CAU) which have been reported in previous studies [14, 15]. The optimal model orders for these four regions are all 1 for MCI patients while 0 for normal controls.

Performance of the proposed method was evaluated using linear support vector machine (SVM) with leave-one-out cross-validation due to limited sample size. It is noteworthy that the feature selection step was performed solely based on the training subjects, without including any information from the testing subjects. MCI identification performance of the proposed method with combined model orders $(q=0$ and $q=1$ ) was compared with (1) the effective connectivity with MAR model order $q=1$, (2) the effective connectivity with MAR model order $q=0$, and (3) the nondirectional Pearson correlation-based functional connectivity. As shown in Table 2, the proposed method yields the best accuracy of $91.9 \%$, which is at least $5.4 \%$ higher than the Pearson correlation-based and the effective connectivity-based methods. The default MAR model order $(q=1)$ based effective connectivity performed slightly inferior to other compared methods with an accuracy of $83.8 \%$. The proposed method also shows an area of 0.903 under the receiver operating characteristic curve (AUC), indicating its excellent generalization capability. Receiver operating characteristic (ROC) curves of all comparison methods are shown in Fig. 1.

The top twelve effective connectivities, which were selected in feature selection step using the training subjects, are listed together with their relationships in Table 3 . Graphical illustration of these selected connectivities is provided in Fig. 2. PCG, an important component in default mode network (DMN), is causally influenced by the 
hippocampus gyrus (HIP) and middle temporal gyrus (MTG), and is correlated with the supramarginal gyrus (SMG), inferior temporal gyrus (ITG), and precuneus (PCNU). DMN is preferentially activated when individuals engage in internal tasks including memories, and gauging others' perspectives. Inferior parietal lobule (IPL) and SMG are important DMN components that involve in the perception of emotions [16]. HIP, which belongs to the limbic system, plays important roles in the consolidation of information from short-term to long-term memory and spatial navigation [17].

Table 1. Classification performance of all comparison methods. $(\mathrm{ACC}=$ accuracy; $\mathrm{CI}=95 \%$ confidence intervals of AUC values)

\begin{tabular}{|l|c|c|c|}
\hline Approach & ACC (\%) & AUC & CI \\
\hline Pearson correlation coefficients & 86.49 & 0.8630 & $0.6821 \sim 0.8325$ \\
\hline MAR model with the default order $q=0$ & 86.49 & 0.8100 & $0.6772 \sim 0.8224$ \\
\hline MAR model with the default order $q=1$ & 83.78 & 0.7867 & $0.6017 \sim 0.7947$ \\
\hline $\begin{array}{l}\text { MAR model with optimal orders } \boldsymbol{q}=\mathbf{0} \\
\text { and } \boldsymbol{q}=\mathbf{1}\end{array}$ & $\mathbf{9 1 . 8 9}$ & $\mathbf{0 . 9 0 3 3}$ & $\mathbf{0 . 7 0 6 2} \sim \mathbf{0 . 8 6 1 4}$ \\
\hline
\end{tabular}

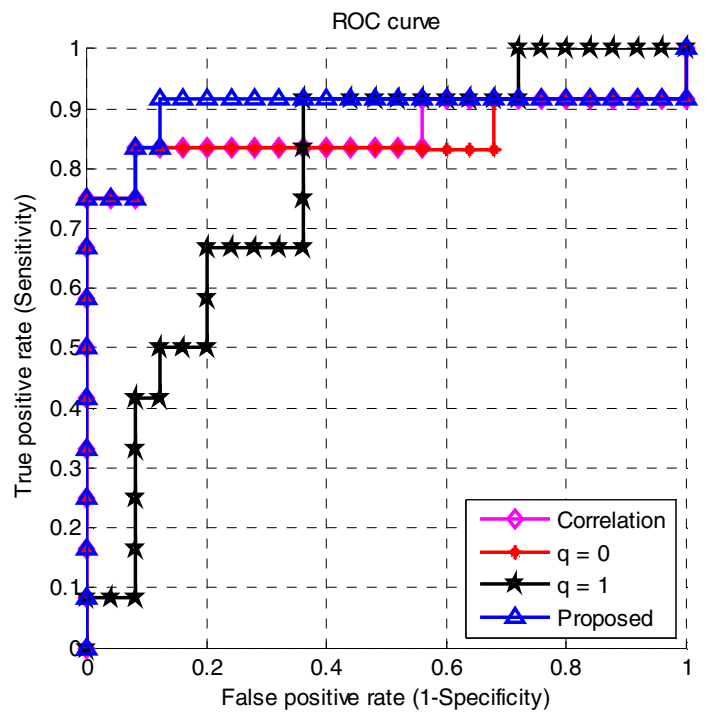

Fig. 1. ROC curves for all comparison methods 
PCNU is an important region that involves in episodic memory [18]. The causal and correlation connectivity abnormalities of these brain regions cause decline in cognitive functioning in MCI and AD subjects [19].

\section{Conclusions}

In this paper, we present a novel approach to infer effective connectivity from RfMRI data using the optimal sparse MAR modeling. The optimal model order is determined based on the MAR model order distributions of each ROI with minimum mean square error. Four brain regions in MCI group are causally influenced by other regions while they are correlated with other regions in control group. An OLS algorithm is incorporated in MAR model to remove possible spurious effective connections. Effective connectivity networks, which are inferred using the optimal model orders, are applied to MCI identification. Promising results obtained indicate that the disruptions of causal relationships among brain regions can be used to improve neurological disorder identification performance.

Table 2. Top 12 effective connectivities selected using the proposed classification framework. $(\longleftarrow$ : direction of causal influence; $\longleftrightarrow$ : correlation; CAU: Caudate; HIP: Hippocampus; IPL: Inferior parietal lobule; ITG: Inferior temporal gyrus; LING: Lingual gyrus; MCG: Middle cingulate gyrus; MTG: Middle temporal gyrus; PCG: Posterior cingulate gyrus; PCUN: Precuneus; SMG: Supramarginal gyrus; R: right; L: left)

\begin{tabular}{|c|c|c|}
\hline $\begin{array}{l}\text { Selected } \\
\text { ROIs }\end{array}$ & Direction of effective connectivity & Connected ROIs \\
\hline PCG.R & 4 & HIP.L \\
\hline PCG.R & $\longleftrightarrow$ & SMG.R \\
\hline MCG.L & $\longleftrightarrow$ & MCG.R \\
\hline PCG.R & $\longleftrightarrow$ & PCG.L \\
\hline MCG.L & 4 & ITG.L \\
\hline PCG.R & $\longleftrightarrow$ & ITG.L \\
\hline LING.L & 4 & LING.L \\
\hline MCG.L & $\longleftrightarrow$ & IPL.L \\
\hline PCG.R & 4 & SMG.L \\
\hline PCG.R & $\longleftarrow$ & MTG.L \\
\hline PCG.R & $\longleftrightarrow$ & PCUN.L \\
\hline CAU.L & $\longleftrightarrow$ & CAU.R \\
\hline
\end{tabular}




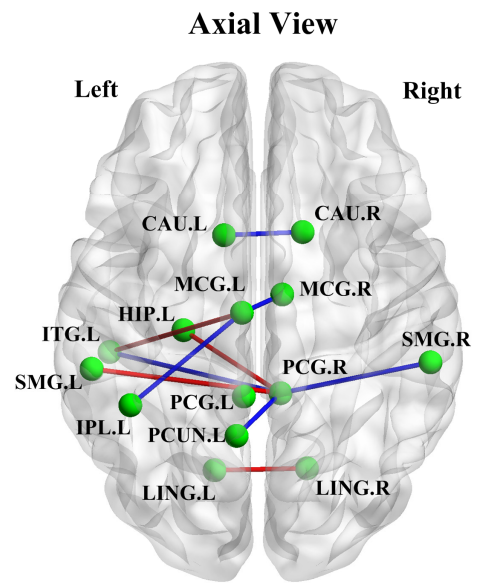

\section{Coronal View}

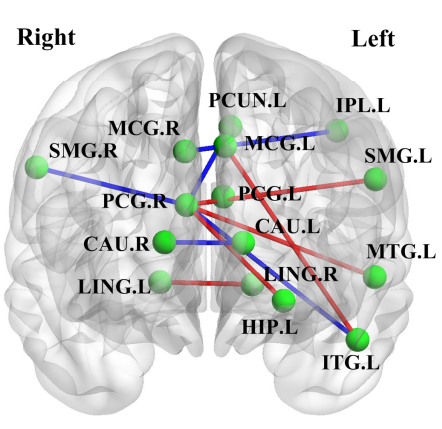

Fig. 2. Most discriminative effective connectivities selected for MCI classification. (CAU: Caudate; HIP: Hippocampus; IPL: Inferior parietal lobule; ITG: Inferior temporal gyrus; LING: Lingual gyrus; MCG: Middle cingulate gyrus; MTG: Middle temporal gyrus; PCG: Posterior cingulate gyrus; PCUN: Precuneus; SMG: Supramarginal gyrus; L: left; R: Right; Green: ROI; Red: Causal relationship; Blue: Correlation relationship)

\section{References}

1. Petersen, R.C., Smith, G.E., Waring, S.C., Ivnik, R.J., Tangalos, E.G., Kokmen, E.: Mild cognitive impairment - Clinical characterization and outcome. Arch. Neurol. 56, 303-308 (1999)

2. Friston, K.: Causal Modelling and Brain Connectivity in Functional Magnetic Resonance Imaging. PLoS Biol. 7, e1000033 (2009)

3. Granger, C.W.J.: Investigating Causal Relations by Econometric Models and CrossSpectral Methods. Econometrica 37, 414-420 (1969)

4. Rajapakse, J.C., Zhou, J.: Learning effective brain connectivity with dynamic Bayesian networks. Neuroimage 37, 749-760 (2007)

5. Tzourio-Mazoyer, N., Landeau, B., Papathanassiou, D., Crivello, F., Etard, O., Delcroix, N., Mazoyer, B., Joliot, M.: Automated anatomical labeling of activations in SPM using a macroscopic anatomical parcellation of the MNI MRI single-subject brain. Neuroimage 15, 273-289 (2002)

6. Murphy, K., Birn, R.M., Handwerker, D.A., Jones, T.B., Bandettini, P.A.: The impact of global signal regression on resting state correlations: Are anti-correlated networks introduced? Neuroimage 44, 893-905 (2009)

7. Goebel, R., Roebroeck, A., Kim, D.S., Formisano, E.: Investigating directed cortical interactions in time-resolved fMRI data using vector autoregressive modeling and Granger causality mapping. Magn. Reson. Imaging 21, 1251-1261 (2003)

8. Harrison, L., Penny, W.D., Friston, K.: Multivariate autoregressive modeling of fMRI time series. Neuroimage 19, 1477-1491 (2003)

9. Liao, W., Ding, J.D., Marinazzo, D., Xu, Q., Wang, Z., Yuan, C., Zhang, Z., Lu, G., Chen, H.: Small-world directed networks in the human brain: Multivariate Granger causality analysis of resting-state fMRI. Neuroimage 54, 2683-2694 (2011) 
10. Wang, K., Liang, M., Wang, L., Tian, L., Zhang, X., Li, K., Jiang, T.: Altered functional connectivity in early Alzheimer's disease: A resting-state fMRI study. Hum. Brain Mapp. 28, 967-978 (2007)

11. Billings, S.A., Wei, H.-L.: Sparse model identification using a forward orthogonal regression algorithm aided by mutual information. IEEE T. Neural Networ. 18, 306-310 (2007)

12. Schwarz, G.: Estimating Dimension of A Model. Ann. Stat. 6, 461-464 (1978)

13. Guyon, I., Weston, J., Barnhill, S., Vapnik, V.: Gene selection for cancer classification using support vector machines. Machine Learning 46, 389-422 (2002)

14. Greicius, M.D., Krasnow, B., Reiss, A.L., Menon, V.: Functional connectivity in the resting brain: a network analysis of the default mode hypothesis. Proceedings of the National Academy of Sciences of the United States of America 100, 253-258 (2003)

15. Chetelat, G., Desgranges, B., de la Sayette, V., Viader, F., Eustache, F., Baron, J.C.: Mapping gray matter loss with voxel-based morphometry in mild cognitive impairment. Neuroreport 13, 1939-1943 (2002)

16. Whitfield-Gabrieli, S., Ford, J.M.: Default mode network activity and connectivity in psychopathology. Annu. Rev. Clin. Psychol. 8, 49-76 (2012)

17. Squire, L.R.: The legacy of patient H.M. for neuroscience. Neuron 61, 6-9 (2009)

18. Cavanna, A.E., Trimble, M.R.: The precuneus: a review of its functional anatomy and behavioural correlates. Brain 129, 564-583 (2006)

19. Miao, X., Wu, X., Li, R., Chen, K., Yao, L.: Altered Connectivity Pattern of Hubs in Default-Mode Network with Alzheimer's Disease: An Granger Causality Modeling Approach. PLoS ONE 6, e25546 (2011) 